\title{
NATURE GENETIQUE DES MUTANTS A DEFICIENCE RESPIRATOIRE DE LA SOUCHE B-II DE LA LEVURE DE BOULANGERIE
}

\author{
SHIH-YI CHEN, BORIS EPHRUSSI et HELENE HOTTINGUER \\ Institut de Génétique du C.N.R.S. et Institut de Biologie physico-chimique, Paris
}

\section{INTRODUCTION}

Received 30.vi.50

Toute population de levure de boulangerie (Saccharomyces cerevisia) contient un certain nombre de cellules mutantes ("petites") qui, placées à la surface d'un milieu nutritif solide, donnent naissance à des colonies naines (Ephrussi, Hottinguer et Chimènes, 1949). La vitesse de croissance réduite de celles-ci est due à l'incapacité des levures mutantes d'utiliser le glucose par la voie respiratoire, de sorte que, même en aérobiose, elles fermentent le sucre au lieu de l'oxyder (Tavlitzki, r949; Slonimski, r949). La déficience respiratoire est, à son tour, la conséquence de l'absence de deux ferments respiratoires essentiels, la cytochrome-oxydase et la succino-déhydrase, fait établi à la fois par l'étude de l'activité enzymatique et par l'examen spectroscopique des cellules mutantes (Slonimski et Ephrussi, 1949).

La nature génétique de la mutation "petite colonie" a été étudiée sur la levure américaine "Yeast Foam" (Y.F.) de $S$. cerevisia (Ephrussi, Hottinguer et Tavlitzki, 1949). Les croisements entre une souche normale et une souche mutante, suivis de quatre backcross à la forme mutante, ont constamment donné naissance à des lignées normales; le caractère mutant n'est réapparu que dans 5 spores sur un total de 596 spores étudiées. L'analyse de ces résultats a conduit à la conclusion qu'ils ne sont compatibles avec un mécanisme mendélien que si l'on admet que les formes normale et mutante diffèrent par un nombre élevé de gènes $(>6$ à 20$)$. Mais une telle interprétation est en contradiction avec la fréquence élevée des mutations spontanées trouvée par ailleurs (Ephrussi, L'Héritier et Hottinguer, 1949). Il en a été déduit que la mutation est probablement de nature cytoplasmique. Enfin, l'ensemble des observations sur les mutants "petite colonie" et des données relatives au processus de mutation a conduit à l'hypothèse que les mutants en question diffèrent de la forme normale par la perte de particules cytoplasmiques autoreproductibles, essentielles à la synthèse d'un groupe d'enzymes respiratoires (l.c.).

Le mutant $\left(\mathrm{I}-\mathrm{A}_{2}\right)$ de Yeast Foam dont l'étude vient d'être résumée est un mutant apparu au cours de la reproduction végétative de la levure. Des mutants de cette sorte apparaissent plus fréquemment encore dans une autre souche de levure de la même espèce, la souche 


\section{$33^{8}$ SHIH-YI CHEN, B. EPHRUSSI AND HELENE HOTTINGUER}

"Boulangerie II " (B-II), et tout porte à croire que le mécanisme de leur formation est analogue à celui révélé par l'étude des mutants de Yeast Foam, c'est-à-dire que la mutation porte sur un facteur cytoplasmique. Mais chez B-II on observe de plus que des mutants à propriétés analogues apparaissent souvent à la suite de la méiose : des ascopores isolés donnent fréquemment d'emblée des clones composés uniquement de cellules du type mutant. Les chiffres du tableau I permettent de constater que 44 pour cent des spores de

TABLEAU I

Comparaison du taux de germination des ascopores et de la fréquence des spores mutantes dans les souches B-II et Y.F.

\begin{tabular}{|c|c|c|c|c|}
\hline \multirow{2}{*}{\multicolumn{2}{|c|}{ Souches }} & \multicolumn{3}{|c|}{ Nombre d'ascospores } \\
\hline & & isolées & germées & mutantes \\
\hline B-II & . & 596 & 27 & I 2 \\
\hline Y.F. & $\cdot$ & I 12 & 85 & 4 \\
\hline
\end{tabular}

B-II appartiennent à cette catégorie. Le taux de germination des spores de cette souche est si bas que nous n'avons jamais observé la germination des quatre spores d'un même asque et nous ignorons par conséquent le type de ségrégation qui s'y produit. Mais la fréquence totale des spores mutantes (44 pour cent) est suffisamment proche de $I / 2$ pour qu'il nous ait paru légitime de nous demander si le caractère mutant n'est pas contrôlé ici par un gène mendélien pour lequel la souche B-II serait hétérozygote, et si l'étude comparée des mutants prenant naissance dans les asques (" petites ségrégantes") et des mutants apparaissant au cours de la reproduction végétative ("petites végétatives") n'était pas susceptible de révéler l'existence d'interactions entre facteurs géniques et cytoplasmiques.

Le présent travail a été entrepris dans le dessein de répondre à ces questions.*

\section{MATERIEL ET METHODES}

(i) Toutes les lignées de levures utilisées dans ce travail ont pour origine deux souches de Saccharomyces cerevisia :

"Boulangerie II" qui provient de la collection du Laboratoire des Fermentations de l'Institut Pasteur de Paris ;

"Yeast Foam," levure de boulangerie américaine, que nous devons à l'obligeance du Professeur $\varnothing$. Winge.

Les souches B-I 5 et B-26 sont des lignées monosporiques haploïdes isolées à partir d'asques de B-II. B-I5 est une "grande" de signe

* Une partie des résultats a fait l'objet de la thèse de Doctorat de S. Y. Chen (1950), présentée à l'Université de Paris. 
$(+)$, B-26 une " petite ségrégante" de signe (-). La souche B-r 5-p4 est une "petite végétative" isolée à partir de B-I 5 et est, comme elle, de signe $(+)$.

La souche I- $\mathrm{A}_{2}$ est une "petite végétative" haploïde dérivée de Yeast Foam. Son origine a été décrite par Ephrussi, Hottinguer et Tavlitzki (1949). Nous rappellerons qu'elle est de signe ( $\rightarrow$ ) et qu'elle porte le gène $a$ (exigence de l'adénine ; pigment rouge).

Toutes les autres souches qui seront mentionnées dérivent des croisements des précédentes.

(ii) Les milieux de culture et de sporulation sont les mêmes que ceux utilisés par Ephrussi, Hottinguer et Tavlitzki (r949). Il en est de même de la technique des croisements.

(iii) Le diagnostic différentiel des "grandes" et des "petites" peut être fait par trois méthodes : réaction colorée de l'indophénoloxydase ("Nadi "), détermination du QR, examen spectroscopique. Toutes les trois ont été appliquées à l'étude des souches mentionnées en (i). Par la suite, nous nous sommes limités au test du Nadi, plus simple et plus économique, effectué selon la méthode de Keilin (1929).

Dans ce travail, la réaction du Nadi nous servira à déterminer la présence de l'indophénoloxydase dans des clones monosporiques d'une part, et dans les cellules diploïdes formées à la suite de croisements de clones haploïdes, de l'autre. Cette deuxième utilisation appelle la remarque suivante. Lorsqu'on fait dans un tube un croisement en masse selon la méthode des Lindegren, ce tube contient au bout de 24 heures un mélange de cellules haploïdes, de figures de copulation et de cellules diploïdes. Les cellules diploïdes possédant un fort avantage sélectif sur les haploïdes, nous effectuons au moins 4 repiquages de la culture afin de l'enrichir en diploïdes et d'éliminer les haploïdes avant de la soumettre au test du Nadi. Gette technique est quelquefois remplacée par l'isolement direct, à l'aide du micromanipulateur, de cellules diploïdes.

La réaction du Nadi est effectuée macroscopiquement sur la suspension de levure lavée.

\section{ANALYSE GENETIQUE DES MUTANTS "PETITE COLONIE"}

(a) Démonstration d'une différence génétique entre "petites végétatives" et "petites ségrégantes." Réapparition de la forme normale dans les croisements des deux types de "petites."

L'existence d'une différence génétique entre "petites végétatives" et " petites ségrégantes" est immédiatement révélée par le croisement de ces deux formes avec une même levure normale.

Le croisement (I) de la "petite végétative" I- $\mathrm{A}_{2}$ (portant le gène a) avec la "grande" B-r 5 (qui porte le gène $A$ ) fournit des cultures diploïdes Nadi positives. I4 asques formés par celles-ci ont été analysés. Ils contenaient tous 2 spores $A$ et 2 spores $a$. D'un autre côté, toutes les 56 spores ont donné naissance à des clones Nadi positifs. 
Ce croisement fournit donc une $F_{1}$ entièrement constituée par des "grandes." Le caractère mutant disparaît ici comme dans les croisements étudiés précédemment (l.c.).

Le croisement (53) de la même "grande" B-I5 avec la " petite ségrégante " B-26 fournit un résultat différent. Les diploïdes hybrides sont encore une fois Nadi positifs, mais, dans ce cas, le caractère mutant réapparait en $F_{1}$. Les 32 asques analysés ont montré une ségrégation régulière en 2 " grandes" : 2 " petites."

Ainsi, les croisements d'une même " grande" avec deux " petites" différentes, l'une "végétative," l'autre " ségrégante," fournissent.des résultats différents. Dans le premier cas, le caractère mutant ne réapparait pas dans les asques de la $F_{1}$. Le second croisement est, au contraire, suivi d'une ségrégation mendélienne régulière. Il en ressort qu'un même phénotype mutant peut reposer sur des bases génétiques différentes. Chez la "petite ségrégante," le caractère mutant est sous la dépendance d'un gène mendélien.

La comparaison qui vient d'être faite porte cependant sur deux "petites" qui diffèrent en outre par la souche d'origine. Afin de la reproduire sans sortir des limites d'une souche, nous avons isolé, a partir de la souche B-I5, la "petite végétative" B-I5-p4. Le résultat du croisement (53) entre une "grande" et une "petite ségrégante" de la souche "Boulangerie II " étant déjà établi, et le croisement entre la même " grande" B-I 5 et la " petite végétative" B-I5-p4 étant impossible (puisque ces deux souches sont du même " signe" "), nous avons effectué le seul croisement possible (24) : " petite ségrégante " B-26 $\times$ " petite végétative" B-I 5-p4. La culture diploïde obtenue était Nadi positive et l'analyse de I 5 asques a montré qu'ils contenaient tous 2 "grandes" et 2 " petites."

Cette expérience apporte, on le voit, un résultat nouveau : le croisement de deux "petites" fournit des asques dans lesquels on observe régulièrement une ségrégation simple en 2 "grandes" : 2 " petites." Ce résultat confirme d'une façon indiscutable la conclusion précédente quant à la différence génétique entre " petites végétatives" et " petites ségrégantes." En outre, elle apporte une justification à la désignation par des termes différents de ces deux sortes de mutants qui présentent le même phénotype et ne diffèrent que par leur mode d'origine.

(b) Essai d'interprétation mendélienne des résultats précédents

Les résultats qui viennent d'être décrits peuvent être représentés par le schéma suivant :

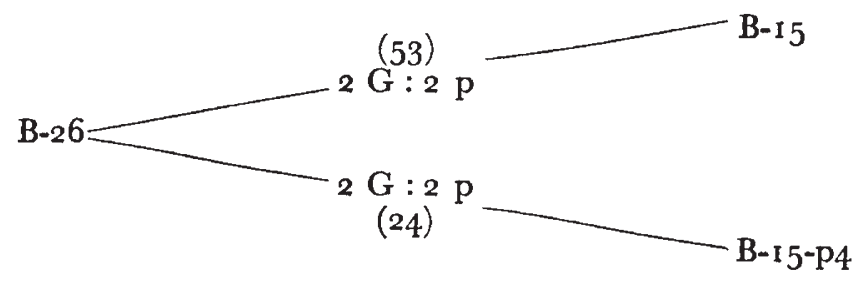


qui met en évidence des relations à première vue paradoxales et dont l'interprétation en termes mendéliens s'avère difficile.

Le croisement 53 conduit à une ségrégation monofactorielle typique : 2 " grandes" : 2 " petites." Comme l'examen des cellules diploïdes qui donnent naissance à ces asques montre que le caractère normal est dominant (les cultures diploïdes sont Nadi positives), nous pouvons en conclure que la souche B-i 5 porte un gène dominant $(R)$ et la souche B-26 son allélomorphe récessif $(r)$. D'un autre côté, le croisement (24) conduit également à la formation de diploïdes Nadi positifs et d'asques à ségrégation en 2 "grandes" : 2 " petites." Le type de ségrégation semble donc indiquer que les souches B-26 et B-I 5-p4 diffèrent aussi par un seul gène, mais ceci est incompatible avec le fait qu'ici le croisement de deux " petites " redonne des formes normales. La réapparition de celles-ci exige, semble-t-il, l'intervention d'au moins deux gènes non allélomorphes.

On peut cependant admettre que la ségrégation $2: 2$ observée dans ce dernier cas n'est monofactorielle qu'en apparence et que nous sommes en présence d'une interaction de deux gènes telle que le phénotype normal est déterminé aussi bien par la présence simultanée de deux dominants que par celle de deux récessifs non allélomorphes. Supposons, comme précédemment, que la "petite ségrégante " B-26 diffère de la " grande " B-I 5 par un seul gène, dont elle porte la forme récessive $r$. Supposons, d'autre part, que la souche " petite végétative" B-I5-p4 résulte de la mutation récessive à un autre locus, soit $B$. Les trois souches étudiées ont alors les génotypes :

$\begin{array}{llllll}\text { Grande } & \cdot & \cdot & \cdot & \mathrm{B}-15 & R B \\ \text { Petite ségrégante } & \cdot & \cdot & \cdot & \mathrm{B}-26 & r B \\ \text { Petite végétative } & \cdot & \cdot & \cdot & \mathrm{B}-15-\mathrm{p}_{4} & R b\end{array}$

Admettons, enfin, que la méiose dans les hybrides comporte un crossing-over obligatoire entre les loci $R$ et $B$. Les résultats des croisements 53 et 24 peuvent alors être représentés comme suit :

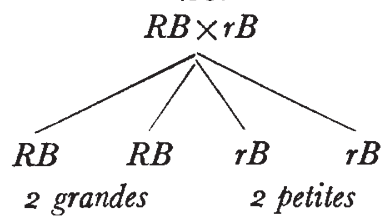

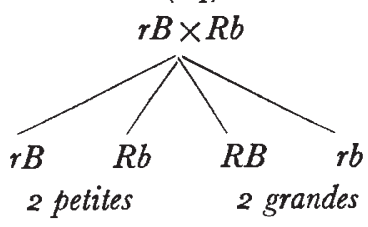

Quoique ce schéma nous ait paru peu vraisemblable, nous l'avons soumis à la vérification expérimentale, car c'est le seul schéma mendélien qui nous ait paru susceptible de rendre compte des observations décrites.

La vérification repose sur le raisonnement suivant : selon le schéma ci-dessus, les asques hybrides du croisement 24 contiennent obligatoirement deux spores "grandes" dont les génotypes sont $R B$ et $r b$, et deux "petites" l'une $r B$, l'autre $R b$. Aussi bien les deux "grandes" 
que les deux "petites" auraient donc des génotypes " complémentaires." Les croisements entre deux " petites " ou deux " grandes" d'un même asque devraient par conséquent conduire :

(i) à des diploïdes " Nadi" positifs

(ii) à des asques montrant une ségrégation 2 "grandes" : 2 "petites."

L'asque No I 3 du croisement 24 nous a permis d'entreprendre cette expérience : ses spores $b$ et $d$ sont des " grandes" de signe opposé et $a$ et $c$ des " petites" de signe opposé. Nous avons donc réalisé les croisements entre les deux "grandes" (63) et entre les deux "petites" (66).

Les résultats du croisement 63 sont les suivants: l'hybride est Nadi positif. Les asques contiennent tous 4 spores normales.

D'un autre côté, l'hybride du croisement 66 est Nadi négatif et ne sporule pas.

Ni la ségrégation observée à la suite du croisement 63 , ni l'absence d'indophénoloxydase dans les cellules diploïdes formées dans le croisement 66 ne sont compatibles avec l'hypothèse que nous avons cherché à vérifier.

\section{(c) Hypothèse invoquant un facteur cytoplasmique}

L'interprétation, en termes mendéliens, des résultats des croisements 24 et 53 s'étant avérée difficile sinon impossible, nous avons formulé une autre hypothèse que nous allons exposer maintenant.

Supposons que la "petite végétative" B-I 5-p4 possède le même génotype $(R)$ que la souche B-I 5 dont elle dérive et résulte de la perte d'un facteur cytoplasmique essentiel à la synthèse des ferments respiratoires. Supposons d'autre part, que ce facteur cytoplasmique, autonome dans sa reproduction, ne fonctionne normalement qu'en présence du gène dominant $R$. Il suffit alors d'admettre que la " petite ségrégante" B-26 porte le gène $r$, pour expliquer l'ensemble des résultats des croisements 53 et 24 :

Les souches B-I 5 et B-26 ne différant que par le couple de gènes $R / r$, leur croisement doit fournir une ségrégation monofactorielle.

D'autre part, le croisement des deux "petites" B-26 et B-I5-p4, dont l'une apporte le gène $R$, l'autre le facteur cytoplasmique inactif, doit aboutir à la reconstitution du système complet (facteur cytoplasmique actif en présence de $R$ ) et à la réapparition de spores à phénotype "grande" dans les asques hybrides, qui montreront ainsi une ségrégation monofactorielle.

Cette interprétation rend donc parfaitement compte des résultats des croisements 53 et 24 , et il est facile de voir qu'elle est également en accord avec ceux des croisements 63 et 66 . En effet, selon cette nouvelle interprétation, les génotypes des deux " petites" de l'asque No I 3 d'une part, ceux des deux " grandes " du même asque, d'autre part, sont pareils; les deux premières portent le gène $r$, les deux 


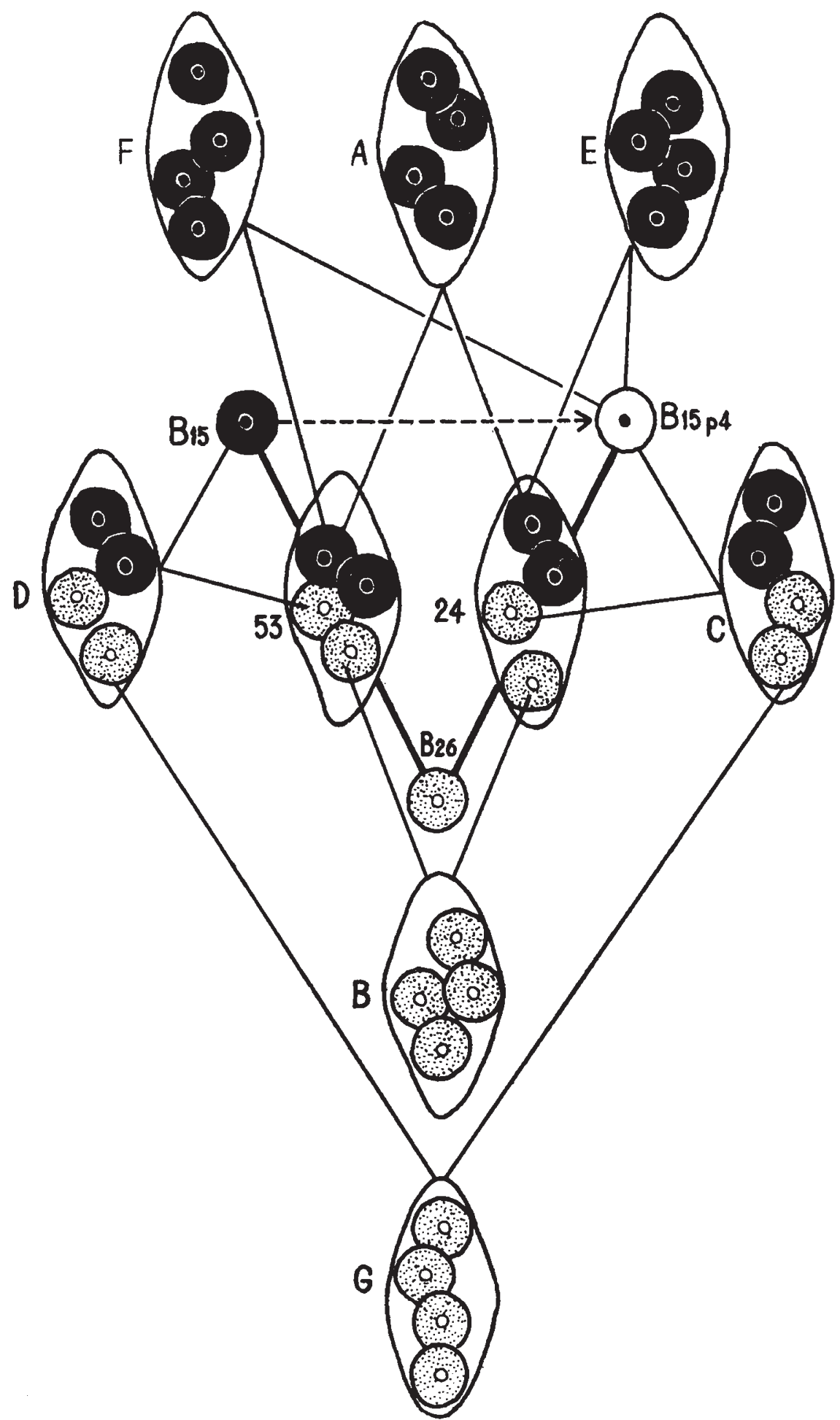

Fig. I.-Schéma des résultats prévus des croisements. En noir: "grandes" (facteur cytoplasmique actif); en grisé : "petites ségrégantes" (facteur cytoplasmique inactif). Noyau noir : gène dominant $R$; noyau blanc: gène récessif $r$. 
seconds, le gène $R$. Le croisement des deux " petites" doit, par conséquent, fournir des diplö̈des $r / r$ Nadi négatifs et le croisement de deux "grandes" des diploïdes $R / R$ Nadi positifs. Etant homozygotes pour $R$, ces diploïdes devraient former des asques à 4 "grandes" : o " petites." C'est ce que nous avons effectivement observé. Les croisements 63 et 66 fournissent ainsi à notre hypothèse une première confirmation.

\section{(d) Vérification de l'hypothèse}

La justesse de l'interprétation proposée peut être vérifiée par des croisements variés. Nous en avons réalisé sept qui nous paraissaient les plus importants et en donnerons maintenant les résultats. La figure I schématise les résultats prévus et permettra de les comparer avec les résultats obtenus.

(i) Croisement entre " grandes" issues des croisements 53 et 24 (fig. I, A). Selon notre interprétation, les "grandes" isolées des asques des croisements 53 et 24 portent le même gène dominant $R$. Elles contiennent par ailleurs le facteur cytoplasmique. Le croisement entre " grandes " provenant des deux croisements doit, par conséquent, fournir des diploïdes Nadi positifs et des asques contenant 4 " grandes" : o " petites."

Deux croisements (200-20I) de ce type ont fourni des diploïdes positifs à la réaction du Nadi. L'analyse de 9 asques a fourni des résultats conformes à la prévision (4 "grandes": o "petites").

(ii) Croisement entre "petites" issues des croisements 53 et 24 (fig. I, B). De même que les " grandes," les " petites issues des deux croisements 53 et 24 doivent, selon notre interprétation, avoir des génotypes identiques $(r)$. Le croisement entre ces " petites" doit, par conséquent, produire des diploïdes Nadi négatifs qui ne pourront former que des spores mutantes.

Quinze croisements de ce type ont été réalisés (136-1 $37,145^{-1} 5^{2}$, I60-16I, 202-204). Conformément à la prévision, tous ont fourni des diploïdes Nadi négatifs. Aucun de ceux-ci n'a malheureusement sporule.*

(iii) Backcross d'une "petite" issue du croisement 24 d la "petite végétative" B-15-p4 (fig. I, G). Les " petites" issues du croisement 24 sont, selon notre hypothèse, identiques à leur parent B-26 dont ils ont reçu à la fois le récessif $r$ et le facteur cytoplasmique. Le backcross de ces "petites" à leur parent B-I 5-p4 doit donc fournir un résultat semblable à celui du croisement 24 .

Les résultats de l'expérience (croisements 75 et 77 ) sont conformes aux prévisions : les diploïdes produits sont Nadi positifs et l'analyse de 18 asques a montré dans tous une ségrégation $2: 2$.

(iv) Backcross d'une "petite" issue du croisement 53 à la "grande" B-I 5 (fig. I, D). La constitution d'une telle "petite" étant, selon

* Ceci est conforme à l'observation que les diploïdes Nadi négatifs ne sporulent jamais (Ephrussi, Hottinguer et Tavlitzki, I949). 
notre interprétation, semblable à celle de la " petite" B-26, ce backcross est strictement équivalent au croisement original (53) et doit fournir un résultat identique.

Conformément à la prévision, le croisement (198-199) a produit des diploïdes Nadi positifs et la ségrégation 2 " grandes" : 2 " petites" a été observée dans 20 asques parmi les $2 \mathrm{I}$ analysés. Toutes les 4 spores du 2 I ème asque étaient "petites."

(v) Backcross d'une "grande" issue du croisement 24 à la "petite végétative" B-I 5-p4 (fig. I, E). Selon notre hypothèse, une telle "grande" tient le gène $R$ du parent B-I5-p4 et le facteur cytoplasmique de l'autre, la "petite ségrégante" B-26. Le backcross à la " petite végétative" $\mathrm{B}$-I 5 -p4, dont le génotype est également $R$, doit fournir des diploïdes possédant le facteur cytoplasmique actif, donc Nadi positifs. Toutes les spores formées par les asques $F_{1}$ contiendront à la fois le gène dominant $R$ et le facteur cytoplasmique. Les asques contiendront donc 4 " grandes."

Le croisement (69) a fourni effectivement une culture Nadi positive. Parmi I 4 asques analysés, I I ont donné le résultat prévu ; trois autres ont, au contraire, montré une ségrégation imprévue en 3 "grande" : I " petite."

(vi) Croisement entre "grande" issue du croisement 53 et la "petite végétative" B-I 5-p4 (fig. I, F). Une telle "grande" tient le gène dominant $r$ du parent " grande" B-I5, et ce gène est, par définition, inchangé dans la " petite végétative" B-I5-p4 qui ne diffère de B-I 5 que par la perte du facteur cytoplasmique. Le diploïde hybride doit donc posséder le facteur cytoplasmique et être homozygote pour $R$. Il sera par conséquent Nadi positif et produira des asques à 4 "grandes."

Quatre croisements de ce type ont été réalisés (II4, II7-II9). Ils ont tous fourni des cultures diploïdes Nadi positives. 27 asques ont été analysés. Les résultats peuvent être résumés comme suit :

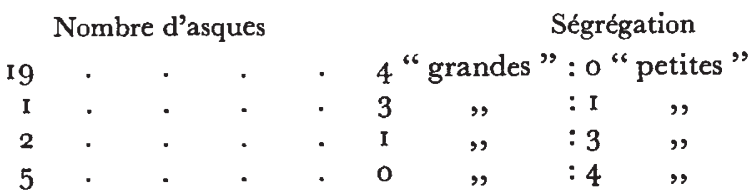

(vii) Croisement entre "petites" issues des backcross décrits en (iii) et (iv) (fig. I, G). Les nombreux croisements entre " petites" issues des croisements 53 et 24 n'ayant pas fourni d'asques (voir ii) nous avons essayé de réaliser un croisement analogue (selon l'hypothèse), en utilisant les " petites" issues des backcross décrits en (iii) et (iv).

Douze croisements (399-4Io) ont été faits dans ce but. Ils ont tous fourni des cultures diploïdes Nadi négatives, mais encore une fois aucune de celles-ci n'a produit d'asques.

(viii) Etude des spores "aberrantes." L'ensemble des résultats des expériences décrites ci-dessus est résumé dans le tableau II (colonnes 
I à 5). L'examen de celui-ci permet de constater ( $\alpha$ ) que l'accord entre prévision et observation est toujours parfait quant aux résultats de la réaction du Nadi exécutée sur les cellules diploïdes hybrides;

TABLEAU 2

Résumé des résultats des croisements

\begin{tabular}{|c|c|c|c|c|c|}
\hline \multirow{2}{*}{ Croisement * } & \multicolumn{2}{|c|}{$\begin{array}{l}\text { Réaction du Nadi des } \\
\text { diploïdes }\end{array}$} & \multicolumn{2}{|c|}{ Ségrégation $\uparrow$} & \multirow{2}{*}{$\begin{array}{c}\text { Nature des spores des } \\
\text { asques aberrants } \ddagger\end{array}$} \\
\hline & prévue & observée & prévue & observée & \\
\hline $\begin{array}{l}53 \\
24 \\
\mathrm{~A} \\
\mathrm{~B} \\
\mathrm{C} \\
\mathrm{D} \\
\mathrm{E} \\
\mathrm{F}\end{array}$ & $\begin{array}{l}+ \\
+ \\
+ \\
+ \\
+ \\
+ \\
+ \\
+\end{array}$ & $\begin{array}{l}+ \\
+ \\
+ \\
+ \\
+ \\
+ \\
+ \\
+\end{array}$ & $\begin{array}{l}2: 2 \\
2: 2 \\
4: 0 \\
0: 4 \\
2: 2 \\
2: 2 \\
4: 0 \\
4: 0\end{array}$ & $\begin{array}{c}3 \text { I }(2: 2) \\
\text { I5 }(2: 2) \\
9(4: 0) \\
\text { pas d'asques } \\
\text { I } 8(2: 2) \\
20(2: 2) \\
\text { I }(0: 4) \\
\text { I }(4: 0) \\
3(3: 1) \\
\text { I9 }(4: 0) \\
\text { I }(3: 1) \\
2(1: 3) \\
5(0: 4) \\
\text { pas d'asques }\end{array}$ & $\begin{array}{c}\ldots \\
\ldots \\
\ldots \\
\ldots \\
\text { (pv pv ps pd) } \\
3(\mathrm{pv}) \\
(\mathrm{pv}) \\
6(\mathrm{pv}) \\
20(\mathrm{pv}) \\
.\end{array}$ \\
\hline
\end{tabular}

* Les numéros indiqués ici correspondent à ceux de la figure I.

† Le premier chiffre donne le nombre de "grandes," le deuxième celui des " petites." ¥ pv "petite végétative"; ps " petite ségrégante"; pd "petite double."

$(\beta)$ qu'à côté des asques du type prévu, d'autres ont été trouvés, dans trois des sept croisements, qui montrent des ségrégations imprévues et que nous allons qualifier " $d$ 'aberrants."

Etant donné que tous les asques aberrants contiennent un excés de spores mutantes, l'hypothèse la plus simple, susceptible de rendre compte, dans le cadre de l'interprétation adoptée, de leur apparition, consiste à admettre qu'elles sont dues à la perte du facteur cytoplasmique postulé. Les "petites" spores surnuméraires, quoique apparues à la suite de la méïose, seraient essentiellement ce que nous avons appelé des " petites végétatives." Elles contiendraient donc toutes le gène dominant $R$.

$\mathrm{La}$ vérification de cette hypothèse a été entreprise de la façon suivante. Le clone dont on cherche à déterminer la nature est croisé, par la méthode de Lindegren : (a) avec un clone standard de "petites végétatives"; $(b)$ avec un clone standard de " petites ségrégantes."* Les cellules diploïdes formées sont soumises au test du Nadi.

Si le croisement $(a)$ donne un test positif et $(b)$ un test négatif, le clone examiné est une " petite ségrégante" $(r)$, puisque la " petite

* Nous avons pris comme clones standard de " petites végétatives" de signe opposé, les souches $1-\mathrm{A}_{8}$ et B-15-p4 mentionnées plus haut. Pour standards de " petites ségrégantes ?" nous avons choisi deux "petites ségrégantes" (19-a et 19-c) d'un meme asque du croisement 53 . 
végétative" avec laquelle il a été croisé était dépourvue de facteur cytoplasmique. Il a donc dû apporter le facteur cytoplasmique. $\mathrm{Si}$, au contraire, le croisement (a) est Nadi négatif et le croisement (b) Nadi positif, le clone examiné est classé comme " petite végétative " puisqu'il a dû apporter le gène dominant $R$ qui manquait à la " petite ségrégante" avec laquelle il a été croisé.

Notons incidemment qu'une troisième possibilité existe également : une levure pourrait à la fois être dépourvue de facteur cytoplasmique et porter le gène récessif $r$. Dans ce cas, les deux croisements $(a)$ et $(b)$ donneraient des résultats négatifs. Nous appellerons ce type de mutant "petite double."

La méthode que nous venons d'indiquer a été appliquée à tous les clones mutants provenant des asques aberrants. Les résultats du test sont donnés par la dernière colonne du tableau 2. On voit que toutes les spores exceptionnelles résultent de la perte du facteur cytoplasmique et que la ségrégation génique dans tous les asques aberrants est conforme à la prévision.

\section{DISCUSSION}

Comme il a été dit dans l'Introduction, le présent travail a été entrepris avec l'espoir de mettre en évidence une interaction entre le génome et le facteur cytoplasmique dont la présence dans les cellules de la levure est indispensable à la synthèse de certains ferments respiratoires.

Cet espoir n'a pas été déçu.

(a) Notre travail a tout d'abord apporté une nouvelle preuve de l'existence de ce facteur cytoplasmique. Les résultats permettent en effet d'affirmer que la souche "petite végétative" B-I 5-p4 ne diffère de la souche-mère "grande" B-I 5 , que par l'absence d'un facteur cytoplasmique autoreproductible. Nous retrouvons donc, entre ces deux souches de la levure " Boulangerie II," des relations exactement semblables à celles qui ont été mises en évidence entre deux souches de "Yeast Foam" (Ephrussi, Hottinguer et Tavlitzki, 1949).

(b) Dans le travail ci-dessus, nous avons pu pousser l'analyse plus loin. Les croisements de la " petite ségrégante" B-26 avec la "petite végétative " B-I 5-p4 et avec la " grande " B-I 5 ont fourni des résultats qui peuvent être expliqués si l'on admet: $(\alpha)$ que les "petites ségrégantes" diffèrent des "grandes" par un seul gène dont elles contiennent l'allélomorphe récessif; $(\beta)$ qu'elles différent des " petites végétatives " à la fois par ce même gène et par le facteur cytoplasmique qu'elles possèdent (comme les "grandes") et dont les "petites végétatives" sont dépouvures. Une série de croisements entrepris pour vérifier ces hypothèses ont montré que les génotypes observés sont toujours en accord avec ceux postulés; et que les quelques aberrations phénotypiques rencontrées s'expliquent raisonablement par la perte du facteur cytoplasmique. 
Tout se passe donc comme si la synthèse des ferments respiratoires dépendait de la présence simultanée d'un facteur cytoplasmique autoreproductible et d'un gène dominant, en absence duquel le facteur cytoplasmique demeure inactif.

Le facteur cytoplasmique de la levure serait donc "autonome" dans sa reproduction; il dépendrait au contraire du génome dans sa fonction. Ces propriétés le rapprochent singulièrement des chloroplastes des plantes.*

(c) Dans le présent travail, nous avons, pour la première fois, introduit une distinction motivée entre "petites végétatives" et " petites ségrégantes." La " petite" I- $\mathrm{A}_{2}$ de Yeast Foam, étudiée dans le travail cité ci-dessus, appartient de toute évidence à la première de ces catégories. Cela explique la disparition du caractère mutant dans les croisements avec une "grande" et dans les quatre backcross effectués par les auteurs; et sa disparition dans le croisement (I) décrit dans le paragraphe III (a). Tout récemment, nous avons réussi à croiser une " petite ségrégante" (No 53-19, provenant donc du croisement 53 ) de $\mathrm{B}-\mathrm{II}$ avec la souche $\mathrm{I}-\mathrm{A}_{2}$, "petite végétative" de Y.F. Ge croisement est suivi d'une ségrégation régulière 2 "grandes" : 2 " petites," ce qui montre que la souche I-A $\mathrm{A}_{2}$ de Y.F. contient le même gène dominant $R$ que la souche $\mathrm{B}-\mathrm{I} 5 \mathrm{de} \mathrm{B}-\mathrm{II}$. Il est donc évident que le facteur cytoplasmique est, dans les deux souches, soumis à un contrôle génique simple. Ces faits rendent probable la suggestion faite dans l'Introduction, selon laquelle la fréquence plus haute de spores mutantes dans les asques de B-II serait due à ce que cette souche est hétérozygote pour un gène récessif.

(d) Dans ce qui précède, nous avons, à dessein, utilisé le terme "facteur cytoplasmique" qui ne préjuge pas de la nature continue ou discontinue de ce constituant. On se rappelle que l'hypothèse d'un facteur héréditaire extranucléaire chez les levures, introduite par Ephrussi et ses collaborateurs, attribue à ce facteur une structure particulaire (voir Introduction).

Notre travail apporte-t-il des éléments nouveaux à ce problème de la structure du facteur cytoplasmique? Il nous paraît que deux constatations faites au cours des recherches relatées ici plaident en faveur de la nature discontinue, c'est-à-dire particulaire, du facteur cytoplasmique.

G'est d'abord la fréquence élevée des ségrégations " aberrantes," qui s'expliquent, comme nous avons pu le montrer, par la perte du facteur cytoplasmique. Considérons en particulier les asques des types $3:$ I et $1: 3$. On ne voit pas comment un facteur cytoplasmique continu, c'est-à-dire imprégnant d'une façon homogène le cytoplasme de la cellule-mère de l'asque, pourrait se répartir inégalement au cours de la sporogénèse.

* L'interprétation proposée est celle qui nous paraît aujourd'hui la plus vraisemblable.

Nous tenons cependant à souligner qu'une autre interprétation de nos résultats expérimentaux est possible si l'on admet : (1) la dépendance de la reproduction des particules cytoplasmiques du gène $R$; (2) un effet retardé de ce gène ; et (3) un seuil de concentration des particules dans leur action physiologique. 
C'est ensuite, la similarité entre les propriétés de notre facteur cytoplasmique et celles des plastes des plantes vertes.

(e) Le mode d'origine des asques " aberrants" sera discuté dans. un prochain mémoire.

\section{RÉSUME}

I. Dans la souche "Boulangerie II" de S. cerevisia des mutants à déficience respiratoire (" petites") apparaissent souvent, d'une part au cours de la reproduction végétative (" petites végétatives"), et d'autre part à la suite de la méïose (" petites ségrégantes"). La fréquence des spores mutantes est d'environ 44 pour cent. Ce pourcentage est suffisamment proche de $1 / 2$ pour qu'il soit légitime de se demander si la souche n'est pas hétérozygote pour un couple de gènes et si le caractère mutant des " petites ségrégantes" n'est pas sous le contrôle d'un gène mendélien.

2. L'étude des deux croisements possibles entre une levure normale ("grande" B-I5), une "petite végétative" (B-I5-p4) qui en dérive, et une " petite ségrégante" (B-26) a fourni les résultats suivants :

(a) Le croisement de la "grande" B-I5 avec la "petite ségrégante" conduit à la formation de cellules diploïdes normales; les asques formés par celles-ci contiennent 2 "grandes" et 2 " petites " spores.

Chez les "petites ségrégantes," le caractère " petite colonie" est donc dû à un gène mendélien récessif.

(b) Le croisement de la "petite végétative" B-I5-p4 avec la " petite ségrégante" B-26 produit des cellules diploïdes normales, et les asques formés par celles-ci contiennent encore une fois 2 "grandes" et 2 "petites" spores.

Dans ce croisement, on obtient, par conséquent, la reconstitution d'une cellule normale par la fusion de deux cellules mutantes. Ceci prouve tout d'abord que des phénotypes identiques peuvent cacher des idiotypes différents; cela montre ensuite que les deux souches croisées doivent différer par quelque chose d'autre que la paire de gènes dont on observe la ségrégation dans les asques $F_{1}$.

3. Ces résultats nous ont conduit à proposer l'interprétation suivante : les " petites végétatives" diffèrent des " grandes" par la perte d'un facteur cytoplasmique nécessaire à la synthèse des ferments respiratoires; les "petites ségrégantes" en diffèrent par un gène récessif en présence duquel le facteur cytoplasmique est inactif.

4. Afin de vérifier cette hypothèse et, en particulier, son corrollaire selon lequel les génotypes des " petites végétatives " et des " grandes" sont identiques, sept types de croisements différents ont été entrepris. L'analyse des asques résultant de ces croisements fournit des génotypes conformes à la prévision. Des phénotypes exceptionnels ont cependant été observés, dont l'étude conduit encore une fois à postuler l'intervention d'un facteur cytoplasmique autoreproductible. 
5. Au total, le présent travail conduit à la conclusion que la synthèse de certains ferments respiratoires requiert la présence simultanée d'un facteur cytoplasmique autoreproductible et d'un gène dominant.

\section{SUMMARY}

I. In the strain "Boulangerie II" of Saccharomyces cerevisia mutants often appear which lack certain respiratory enzymes (" little colony" or "little"). They arise in the course of vegetative reproduction ("vegetative littles") as well as following meiosis ("segregational littles"). The frequency of the mutant spores is about 44 per cent., i.e. so close to $\mathrm{I} / 2$ that the question was raised as to whether the strain is heterozygous for a gene pair, and the mutant character controlled by a mendelian gene.

2. A study of the two crosses possible between a normal clone (" big," B-15), a "vegetative little" (B-15-p4) derived from it, and a " segregational little" (B-26), has given the following results :

(a) The cross of " big " B-I 5 with the "segregational little" B-26 gives rise to normal diploid cells which form asci containing 2 " big" and 2 "little" spores.

Consequently, the character "little" is due to a single mendelian recessive gene in the "segregational littles."

(b) The cross between the "vegetative little" B-I5-p4 and the "segregational little" B-26 also gives normal diploid cells, the asci from which likewise contain 2 " big" and 2 " little" spores.

Thus, in this cross, the fusion of two mutant cells leads to the reconstitution of a normal cell. First of all this shows that the two phenotypically identical cells used in the cross conceal different idiotypes. Secondly it demonstrates that the two strains differ from each other by something other than the gene pair whose segregation takes place in the $F_{1}$ asci.

3. These results have led to the following interpretation: the "vegetative littles" differ from the "bigs" or normals by the loss of a cytoplasmic factor which is necessary for the synthesis of certain respiratory enzymes; the "segregational littles" differ from normals by a single recessive gene in the presence of which the cytoplasmic factor is inactive.

4. Seven different kinds of crosses were undertaken in order to test this hypothesis, and in particular its corollary, according to which the "vegetative littles" and the "bigs" have the same genotypes. The genotypes of the segregants in the asci of these crosses were found to be those predicted by the above hypothesis. However, some exceptional phenotypes were observed, study of which has again led to the postulation of a self-reproducing cytoplasmic factor.

5. As a whole, the present work leads to the conclusion that the synthesis of certain respiratory enzymes in yeast requires the simultaneous presence of a self-reproducing cytoplasmic factor and a dominant gene. 


\section{BIBLIOGRAPHIE}

CHEN, s. Y. 1950. Contribution à l'étude génétique des mutants à déficience respiratoire chez la levure de boulangerie. Thèse, Paris.

EPHRUSSI, B., hotTINGUER, H., ET CHIMENES, A. M. 1949. Action de l'acriflavine sur les levures I. La mutation " petite colonie." Ann. Inst. Pasteur, 76, 35 I-367.

EPHRUSSI, B., HOtTINGUER, H., ET TAVLITZKI, J. 1949. Action de l'acriflavine sur les levures II. Etude génétique du mutant "petite colonie." Ann. Inst. Pasteur, 76, 419-450.

ephrussi, B., L'Heritier, P., et hottinguer, H. 1949. Action de l'acriflavine sur les levures VI. Analyse quantitative de la transformation des populations. Ann. Inst. Pasteur, 77, 64-83.

kEILIN, D. 1929. Cytochromes and respiratory enzymes. P.R.S., B, 104, 206-252.

Lindegren, C. G., ET Lindegren, G. I943. A new method for hybridizing yeast. P.N.A.S., 29, 306-308.

sLONimski, P. P. 1949. Action de l'acriflavine sur les levures IV. Mode d'utilisation du glucose par les mutants " petite colonie." Ann. Inst. Pasteur, 76, 510-530.

SLONimski, P. P., ET EPHRUSSI, B. 1949. Action de lacriflavine sur les levures V. Le système des cytochromes des mutants "petite colonie." Ann. Inst. Pasteur, $77,47-63$.

tavlitzki, J. 1949. Action de l'acriflavine sur les levures III. Étude de la croissance des mutants " petite colonie." Ann. Inst. Pasteur, 76, 497-509. 